\title{
ENSALMO TZELTAL DE 1725
}

\author{
Alejandro Sheseña Hernández \\ Virginia Margarita López Tovilla
}

\section{Introducción}

E n el Archivo Histórico Diocesano de San Cristóbal de Las Casas se conserva un expediente de especial interés histórico. 'Se trata de un auto criminal de 1725 en contra de Sebastián Hernández, indígena tzeltal de Ocosingo, Chiapas, por el delito de "maléfico brujo hechicero de primera clase curandero". ${ }^{2}$ El expediente consta de varios documentos en los cuales se narra de manera detallada no solo el proceso criminal completo sino también el asunto referente a las curiosas maneras cómo Sebastián Hernández desarrollaba su oficio de sanador. De acuerdo con la narración, los procedimientos médicos que practicaba el acusado para sanar a las personas consistían en el uso de hierbas $\mathrm{y}$ en el pronunciamiento de invocaciones y ensalmos "diabólicos" en lengua tzeltal. La transcripción de uno de esos ensalmos (con su traducción al español) es incluido entre los documentos que sustentan el

Alejandro Sheseña Hernández, doctor en Historia por la Universidad Estatal de Voronezh, Rusia. Profesor de Tiempo Completo de la Facultad de Ciencias Sociales de la Universidad Autónoma de Chiapas. Correo electrónico: sesena@ hotmail.com.

Virginia Margarita López Tovilla, Licenciada en Historia por la Universidad Autónoma de Chiapas. Asistente en el Archivo Histórico Diocesano. Correo electrónico: vicky288@hotmail.com. auto criminal, lo cual dota de especial singularidad al expediente ya que da la oportunidad de abordar, a través de una fuente procedente de los propios tzeltales, el asunto de las concepciones indígenas coloniales referentes al origen de las enfermedades y los medios para contrarrestarlas. Este ensalmo será, por ello, el centro de atención del presente comentario.

\section{El proceso judicial}

De acuerdo con los documentos que componen el expediente, el proceso en contra de Sebastián Hernández se desarrolló, en resumen, de la siguiente manera. El 2l de marzo de 1725 compareció ante Esteban Rosales, cura de Ocosingo, el señor Juan Ramírez acusando a Sebastián Hernández de haber "hecho mal" a su sobrino. Este último había caído enfermo de asma después de haber reñido con Sebastián Hernández, hecho que fue interpretado como producto de un maleficio enviado por éste ultimo. Para respaldar aún más su acusación, Juan Ramírez agregó que también un hijo del fiscal de Ocosingo, el señor Antonio de la Cruz, había caído enfermo por obra mágica del acusado. Dada esta implicación, Esteban Rosales citó al siguiente día al fiscal para corroborar la aseveración de Juan Ramírez. El fiscal comentó que no estaba seguro de 
que la enfermedad de su hijo proviniera de Sebastián Hernández. Precisó que en una ocasión él mismo había citado al acusado para cuestionarlo acerca de si en verdad la enfermedad de su hijo había sido producto de un maleficio (pues al igual que el sobrino de Juan Ramírez, el hijo del fiscal también echaba "una babosa por la boca"), a lo que Sebastián Hernández le respondió que "no tenía intención de hacerle nada". El fiscal sin embargo narró varios incidentes criminales y fantásticos en los que el acusado había estado involucrado.

Con estos dos testimonios, Rosales en los siguientes días mandó a aprehender a Sebastián Hernández. Estando ya en prisión, el acusado compareció ante Rosales tres veces en el transcurso de ocho días. Sebastián Hernández se ofreció a curar al sobrino de Juan Ramírez después de confesar que era curandero y que sabía cómo sanar a los hechizados (esto sin embargo sería más adelante negado por él mismo afirmando en su lugar que sólo sabía curar a los que enfermaban de vergüenza). Para ello el acusado en la primera comparecencia proporcionó a Rosales los nombres de las hierbas, los ensalmos en lengua tzeltal con los que curaría al sobrino de Juan Ramírez, y otros datos más. Mucha de esta información fue anotada por el cura de Ocosingo. Con el objeto de recolectar las hierbas correspondientes para la curación, se le permitió a Sebastián Hernández ir a su casa acompañado de custodios. Sin embargo, una vez dentro de su domicilio, y al enterarse de que el sobrino de Juan Ramírez finalmente había muerto, el acusado trató de ahorcarse después de pedir a su madre que se fuera. Los custodios en un principio lo tomaron por muerto pero después, al percatarse de que su corazón latía, hicieron todo lo posible para evitar el suicidio. Todos ellos atestiguarían días después al respecto.

Sebastián Hernández (y su madre) fueron enviados a Ciudad Real de Chiapa el 31 de marzo del mismo año 1725 para que comparecieran ante Don Juan de Santander, Dean de la Santa Iglesia Catedral, Comisario de los Santos Tribunales de la Inquisición, y Juez Eclesiástico y de Testamentos. Los ensalmos y demás información fueron enviados por Rosales a Santander un poco después, el 3 de abril. En Ocosingo la casa de del difunto sobrino de Juan Ramírez fue agredida con piedras; la autoría de este acto se le adjudicó a la esposa de Sebastián Hernández, Jacinta Saraos, a la que también se le acusó de hechicería. En Ciudad Real el acusado negó que fuera brujo y curandero y añadió que había ofrecido falsamente curar al sobrino de Juan Ramírez por miedo a los azotes con los que Rosales lo había amenazado si moría el enfermo. De cualquier forma, Sebastián Hernández fue juzgado por "brujo maléfico de primera clase" y condenado por Santander el 25 de septiembre de 1725 a doscientos azotes dados públicamente por las calles de Ciudad Real y a ser enviado a la prisión de Laguna de Términos para trabajar el resto de su vida. Sebastián Hernández fue notificado de esto el nueve de octubre del mismo año.

\section{El ensalmo}

Como queda dicho en los párrafos anteriores, entre las evidencias que Rosales anexó al expediente criminal, como soporte para las acusaciones, se encuentra un ensalmo en tzeltal proporcionado por el propio acusado. El rezo fue transcrito en su lengua original y traducido al español por el mismo cura de Ocosingo. Rosales además agregó algunos datos más, confesados por Hernández, relativos al contenido de la plegaria. Este ensalmo será citado a continuación iniciando por la transcripción tzeltal y siguiendo después con la traducción española. Los textos, por supuesto, son copiados literalmente, sin embargo, cabe aclarar que Rosales en su transcripción tzeltal pasa por alto distinguir, entre otros aspectos, la diferencia entre los fonemas glotalizados y los no glotalizados, rasgo característico de las lenguas mayas. ${ }^{3}$ Pues bien, el texto tzeltal del ensalmo es el siguiente:

Haat yaotic bai uan utalat at uan? me at uan talemat ta ghun cux otanil? ta olanibal? me at uan talemat ta 
ghun yolil be? ta ghun xchan chucul be? haat yaotic bai uan utalat? me at uan zbulat zti? zcolaalat zti? ta ghuntox ta oxlaghuntox axan yaotic chican ulat talel, ulauacaba ta ztzacau yoc, ta ztzacau zcab, te queze, te qtane, te qpacaue, yaotic ma avunuqueix, ma alecoghuquix, togh hoon cuunix, hoon qta taualix te qtane te queze te qpacaue haxan hoon yaotic yac xochon talel, yac xtal qloquezat yaotic, haat yaotic zacba itzat queremucon yeba itzat queremucon yaotic ma avunuc yaotic hoon cuunix yaotic hoon qlecoghix yaotic te qtane te queze te qpacaue hauc yaotic yeba itzat zacba itzat queremucon, haat yaotic yeba chan chai zacba chan chai queremucon, haat yaotic ghuntox oxlaghuntox pucughat ma avuunuc ma alecogh hoon cuunix haat ghun chacabulux oxlaghun chacabulux haxtuunix te xchaal cabe te xchaal oque zlaanil cab zlaanil oc, avuun yaotic yac xochon, xochonix yaotic ta ztoghol te queze, te qtane, te qpacaue.

A continuación Rosales proporciona la traducción al español:

Aora tu de donde beniste, eres acaso benido de algun descansadero o apeadero, por ventura eres benido de la mitad de algun camino, o de algún camino que se divide en cuatro partes, tu aora de donde beniste, por ventura eres tu la espuma o veneno de la voca de (ghuntox) o de (oxlaghuntox) mas aora se conose que haz benido haz benido a ponerte en las coyonturas de los pies y de sus manos en mi ofisio en mi pecho y en mi postradura aora no es tuyo no es tu quenta verdaderamente es mio basta mi pecho mi ofisio y mi postradura aora entro yo bengo a echarte aora tu aora animal blanco o prieto y muchacho aora lla no es tullo ya es mio es ahora quenta mia mi pecho mi ofisio y mi postradura mas aora yo muchacho animal blanco o prieto tu y yo muchacho anguilla prieta o blanca tu aora (ghuntox) (oxlaghuntox) diablo ya no es tullo ya es mio tu aora (ghun chacabulux) o (oxlaghun chacabulux) es menester aora la asperesa de las manos y pies y la suabidad de las manos y pies por tu causa entro aora, entro aora con mi ofisio mi pecho y postradura.

Y por último agrega la siguiente información:

Aquel Ghuntox y tambien el Oxlaghuntox que estan entre parentesis dice dicho Sebastian Hernandez que es nombre de un indio viejo que estaba antiuamente en un serro el qual enseño a los antiguos y de ai an benido enseñando este modo de curar hasta estos tiempos y lo mismo dice de el Ghun chacabulux y de el Oxlaghun chacabulux que son nombres de otros. El oxlaghuntox es el mismo de que habla el Señor Don Francisco Nuñes en la Diocesanas en el Preambulo en que dice que el que llaman oxlaghuntox le tienen pintado con treze potestades sentado en una cilla con cuernos en la cabeza como carnero P. 9 n. 32.

Las yervas con que cura son estas zaquil chonay, ahcante tzimate, laghante que por no conoserlas no saber como se llaman en castilla no las pongo.

Haze dicho Sebastian Hernandez un chiflido para curar según dice y yo digo que quien sabe explicar estos nombres y sabe todo esto mucho mas sabra.

\section{Las concepciones tzeltales del siglo XVIII con respecto a las enfermedades}

Como se mencionó al inicio del presente artículo, el ensalmo de Sebastián Hernández proporciona invaluable información acerca de las creencias tzeltales del siglo XVIII referentes a las causas de las enfermedades y las maneras de curarlas. Los pormenores contenidos en el ensalmo, aunados a información coincidente y complementaria obtenida de los registros etnográficos modernos, nos permitirán reconstruir determinados detalles, antes ignorados, de las concepciones indígenas coloniales acerca de las enfermedades y de los métodos para curarlas. 
El dato quizá más importante referido en el ensalmo de Sebastián Hernández es el relativo a la participación de ciertas divinidades en el proceso de enfermedadcuración. En particular, el ensalmo menciona a las deidades llamadas Ghun Tox Oxlaghun Tox (1 Tox 13 Tox) y Ghun Chacabulux Oxlaghun Chacabulux (1 Chacabulux 13 Chacabulux). Como bien lo notó Rosales en sus comentarios, 13 Tox es la misma divinidad que Francisco Núñez de la Vega describe como un "demonio" con trece potestades pintado "en silla y con astas en la cabeza, como de carnero" (Núñez de la Vega, 1988: 274).

Fuentes comparativas señalan que los nombres 1 Tox y 13 Tox corresponden en realidad a determinados días en el calendario indígena. Tox es el nombre del sexto día de la veintena tzeltal colonial (Pineda, 1888) y corresponde al día homónimo Tox de la veintena qanjobal, al día Kame ("muerte") de la quiché, y al día Kimi ("muerte") de los antiguos yucatecos (LaFarge y Byers, 1997: 180; LaFarge, 1994: 201). Sin embargo, cabe destacar que en las creencias de los actuales qanjobales, quienes hasta la fecha conservan este sistema calendárico, los nombres de los días son en realidad los nombres de las divinidades que los rigen; estas deidades poseen determinados poderes ambivalentes que pueden incrementarse o disminuirse en su día según el numeral de la trecena calendárica con el que coincidan. Conociendo los valores de estas combinaciones, un adivino experimentado puede fácilmente pronosticar eventos para determinado día (LaFarge, 1994: 204). Entre los quichés estas divinidades de los días están asociadas con altares ubicados en las cimas de determinadas montañas (Tedlock, 2002: 70).

Pues bien, según los qanjobales de Santa Eulalia, la divinidad Tox posee poderes que operan "contra la enfermedad en los caminos" (LaFarge, 1994: 206). Los jacaltecos afirman que Tox también puede actuar "contra los brujos" (LaFarge y Byers, 1997: 166). Para los actuales qanjobales, la divinidad Tox destaca también porque durante su día "puede comenzar a mostrarse alguna enfermedad que nos ataca" (Tejada Buscayrol, 2005). ${ }^{4}$ En combinación con los numerales 1 y 13 justamente, la deidad Tox de los qanjobales trae dinero (LaFarge, 1994: 205); sin embargo, tal combinación entre los quichés señala que una persona enferma irremediablemente morirá (Tedlock, 2002: 97). Los antiguos yucatecos, según el Chilam Balam de Káua, creían que las personas nacidas en un día dominado por Kimi estaban destinadas a ser asesinos (Barrera Vásquez y Rendón, 1989: 91). 1 Tox 13 Tox era, por lo visto, una divinidad ambivalente. Su aparición en el ensalmo en cuestión indica claramente que los tzeltales coloniales compartían las mismas creencias de sus vecinos de Guatemala con respecto a la participación de las divinidades en las enfermedades. Por ello, dada la relación existente entre 1 Tox 13 Tox y los malestares referidos, no es de sorprender que en el ensalmo se asocie a esta divinidad con el mal ocurrido al sobrino de Juan Ramírez. ${ }^{5}$

El ensalmo subraya una especial relación entre 1 Tox 13 Tox y ciertas enfermedades provenientes básicamente de los cruces y centros de caminos (yolilbe, chan chucul be), y también de descansaderos (cux otanil), y paraderos (olanibal). Como hemos visto, también entre los qanjobales la divinidad Tox es vinculada específicamente con las enfermedades "en los caminos" (LaFarge, 1994:206). Dadas estas asociaciones, es posible calificar a 1 Tox 13 Tox como una divinidad especializada en este tipo de enfermedades. Cabe señalar que la insistente alusión de los caminos (y paraderos o descansaderos) en las concepciones indígenas en cuestión se debe a la creencia, encontrada por ejemplo entre los tzotziles actuales, de que el trayecto diario a través de los caminos puede verse marcado por la presencia de ramas embrujadas abandonadas adrede en medio del sendero; pisarlas puede llevar a la adquisición de las enfermedades portadas por esas ramas (Holland, 1978: 139-140; Page Pliego, 2005: 249). Este miedo a los infortunios del camino lo encontramos ya entre los antiguos mayas. En las inscripciones contenidas en 
la vasija K791 se hace referencia a un temible nagual llamado tahn bihil cham (TAN-bi-hi-li CHAM en la transcripción epigráfica), nombre que justamente significa "La Muerte de Medio Camino" (Hoopes and Mora Marín, 2009: 295).

Ahora bien, el ensalmo precisa que el dios 1 Tox 13 Tox está actuando aquí como el causante de la enfermedad del paciente. Afirma que la afección es espuma (bul) o "veneno" (colaal) procedente de la boca de esta divinidad, a la cual denomina con el término pucugh “diablo". Aquí por lo visto se estaba considerando el aspecto destructivo de la divinidad, aquel que anuncia entre los quichés la muerte irremediable de los enfermos. Al respecto de dioses destructivos, es de recordar cómo en el Popol-Vuh son los dioses del sitio llamado Xibalba los encargados de causar afecciones en las personas (Recinos, 1978: 50-51). Los actuales tzotziles creen que hay enfermedades que pueden ser enviadas a la gente por los anheles dueños sobrenaturales de las montañas si así se les pide (Holland, 1978: 124). A juzgar por el ensalmo, la divinidad 1 Tox 13 Tox formaba parte de este conjunto de entidades maléficas de las montañas pues no sólo causaba enfermedad y muerte sino incluso también era, como lo señala la confesión de Sebastián Hernández, dueño sobrenatural de una montaña. Recuérdese que entre los quichés las divinidades de los días están asociadas con altares ubicados justamente en las cimas de determinados cerros (Tedlock, 2002: 70).

Una de las formas que tenía 1 Tox 13 Tox para perjudicar era, como hemos visto, enviando o patrocinando maleficios que pudieran adquirirse en los caminos y los descansaderos. Sin embargo, en el ensalmo se especifica otro tipo de procedimientos. En particular, 1 Tox 13 Tox podía enviar a las personas animales portadores de enfermedades, entre ellos la anguila (chan chai), un pez con forma de serpiente. Núñez de la Vega también menciona que los brujos que el trató gustaban de enviar culebras y ciempiés causante de enfermedades (Núñez de La Vega, 1983: 9). De acuerdo con el ensalmo, 1 Tox 13 Tox enviaba los animales pronunciando palabras pues el término colaal en el tzeltal colonial significa "blasfemia" más que "veneno" (Ara, 1986: 275). Los actuales tzeltales creen que las palabras también pueden causar dolencias (Pitarch, 1996: 241-250). Las anguilas eran enviadas por 1 Tox 13 Tox para que penetraran en la víctima a través de las coyunturas de piernas y brazos (ulauacaba ta ztzacau yoc, ta ztzacau zcab) y pudieran originar el malestar. También los actuales tzotziles piensan que los animales con enfermedades penetran "en las coyunturas de sus huesos, en las conexiones de sus cuerdas" (yech'al bak', yech'al ch'usub) (Holland, 1978: 252). Por lo visto, este tipo de agresiones también eran parte del repertorio de la divinidad 1 Tox 13 Tox. La atención en las coyunturas como punto corporal delicado parece ser, a propósito, una constante en las creencias indígenas relativas a las enfermedades, constante que se remontaría incluso a tiempos prehispánicos. Virginia López Tovilla ha argumentado recientemente que los anillos, ajorcas, pulseras y tobilleras de jade de los antiguos mayas habrían servido justamente para proteger las coyunturas del cuerpo contra maleficios enemigos (gracias a las cualidades mágicas protectoras que en las creencias poseía el jade) (López Tovilla, 2009: 84-86).

Cabe destacar, por último, el otro aspecto presente en 1 Tox 13 Tox: el de sanador. Recuérdese que entre los qanjobales y quiches la deidad Tox (Kami) está relacionada tanto con la muerte del enfermo como también con su curación (LaFarge, 1994: 206; Tedlock, 2002: 97). De manera semejante se daba entre los tzeltales coloniales pues, según la confesión de Sebastián Hernández, 1 Tox 13 Tox era quien en el pasado había enseñado a los ancestros del acusado las técnicas para curar. De acuerdo con los actuales ch'oles de Tila, la deidad de la montaña puede enseñar tanto a dañar con enfermedades como también a curar (Manca, 1995: 229). Tal ambivalencia está ligada a aquella creencia, encontrada por ejemplo entre los tzotziles de hoy, de que cualquier personaje que sabe enviar una enfermedad debe saber también alejarla pues las fuentes del poder 
sobrenatural, sea cual fuere, son las mismas (Holland, 1978: 134, 177; Maurer, 1983: 416, 421).

En este caso la cura del "mal de la anguila" relacionado con 1 Tox 13 Tox consistía, según el ensalmo, en penetrar en la persona (och) para apoderarse de ella y poder expulsar (loquez) a los animales mencionados. La propia existencia del ensalmo señala que la curación requería de la comunicación con la divinidad maléfica para exigirle el retiro del mal, razón que explica el formato de la plegaria en cuestión. ${ }^{6}$ Naturalmente, antes se requería de un diagnóstico de las causas de la enfermedad, requisito que se refleja en el ensalmo cuando Sebastián Hernández pregunta de dónde proviene el mal. Así se acostumbra entre los modernos tzotziles y tzeltales (Holland, 1978: 177; Maurer, 1983: 423-424). ${ }^{7}$ Los ensalmos en general habrían jugado un papel muy importante en los procedimientos médicos de los tzeltales coloniales (Ruz, 1992: 334-335). Según la confesión de Sebastián Hernández, la curación también requería del uso de las plantas llamadas zaquil chonay, ahcante tzimate y laghante en la transcripción de Rosales. Los curanderos tzotziles utilizan plantas cuando desean que éstas absorban el mal del enfermo; al término de la sesión de curación las plantas "infectadas" son abandonadas adrede en medio de algún camino para que alguien más absorba la afección (Holland, 1978: 176). Es posible que Sebastián Hernández se dispusiera a proceder de la misma forma con respecto a este "mal de la anguila". En tal caso, la idea habría sido abandonar en alguna vereda las plantas citadas con el propósito de que finalmente algún caminante, en su falta de precaución, adquiriera el mal de 1 Tox 13 Tox, el señor de las enfermedades "en el camino".

\section{Notas}

${ }^{1}$ Los autores desean agradecer a Rafaela Gómez Morales, encargada del Archivo Histórico Diocesano, por las facilidades prestadas en la consulta y registro del expediente motivo de la presente publicación.
${ }^{2}$ El documento se localiza en la carpeta III A 1 (Asuntos Indígenas-Catequización y Gobierno-Idolatría).

3 La precisión de la transcripción tzeltal y de la traducción española de Rosales fue revisada con auxilio de los diccionarios tzeltales de Ara (1986) y de Slocum, Gerdel y Cruz Aguilar (1999). En este proceso también participó Marceal Méndez Pérez a quien reconocemos su colaboración.

${ }^{4}$ Agradecemos a Mario Tejada Buscayrol por compartir generosamente con nosotros sus notas de campo hechas en la comunidad de Santa Eulalia, Guatemala, en el año 2005.

${ }^{5}$ La deidad 1 Chacabulux 13 Chacabulux debió ser de la misma naturaleza; desgraciadamente no contamos con datos suficientes para abordarla.

6 Pedro Pitarch destaca que uno de los rasgos característicos de los ensalmos tzeltales actuales es la polifonía: el rezador habla a la vez por sí mismo y por todas las divinidades con las que trata (Pitarch Ramón, 1996: 246).

${ }^{7}$ Los ensalmos indígenas en toda el área maya presentan estructuras semejantes según lo podemos constatar en las oraciones yucatecas contenidas en el Ritual de los Bacabes. Los ensalmos yucatecos contenían, entre otros elementos, un diagnóstico y un conjuro precisamente (Arzápalo Marín, 1987: 11), tal como lo notamos en el ensalmo tzeltal objeto del presente artículo.

\section{Bibliografía}

Ara, Fray Domingo de (1986), Vocabulario de lengua tzeldal según el orden de Copanabastla. Editado por Mario Humberto Ruz, México: Universidad Nacional Autónoma de México.

Arzápalo Marín, Ramón (Editor) (1987), El ritual de los bacabes, México: Universidad Nacional Autónoma de México.

Barrera Vásquez, Alfredo y Silvia Rendón (1989), El libro de los libros de Chilam Balam, Mérida: Editorial Dante. 
Holland, William R. (1978), Medicina maya en los altos de Chiapas, México: Instituto Nacional Indigenista.

Hoopes, John, and David Mora Marín (2009), Violent Acts of Curing: Pre-Columbian Metaphors of Birth and Sacrifice in the Diagnosis and Treatment of Illness "Writ Large", in Blood and Beauty: Organized Violence in the Art and Archaeology of Mesoamerica and Central America, Edited by Heather Orr and Rex Koontz. Ideas, Debates, and Perspectives 4, Cotsen Institute of Archaeology Press. pp. 291-330.

LaFarge, Oliver (1994), La costumbre en Santa Eulalia, Guatemala: Ediciones Yaxte'.

LaFarge, Oliver y Douglas Byers (1997), El pueblo del cargador del año, Guatemala: Ediciones Yaxte'.

López Tovilla, Virginia Margarita (2009), Los ornamentos de jade entre los antiguos mayas, Tesis de Licenciatura en Historia, San Cristóbal de Las Casas, México: Universidad Autónoma de Chiapas.

Manca, María Cristina (1995), "De las cuevas hasta el cielo pasando por los colores de las enfermedades", en Anuario $V$ del Instituto de Estudios Indígenas, Tuxtla Gutiérrez: Universidad Autónoma de Chiapas, pp. 223-259.

Maurer, Eugenio (1983), Los tseltales, ipaganos o cristianos?; su religión, isincretismo síntesis?, México: Centro de Estudios Educativos.

Núñez de la Vega, Francisco (1983), "Carta IX Pastoral”, en Boletín 5 del Archivo Histórico Diocesano, San Cristóbal de Las Casas: INAREMAC.
Núñez de la Vega, Francisco (1988), Constituciones diocesanas del obispado de Chiapa, Edición preparada por María de León Cáceres y Mario Humberto Ruz, México: Universidad Nacional Autónoma de México.

Page Pliego, Jaime Tomás (2005), El mandato de los dioses. Etnomedicina entre los tzotziles de Chamula y Chenalhó, Chiapas, México: Universidad Nacional Autónoma de México.

Pineda, Vicente (1888), Historia de las sublevaciones indígenas. Gramática de de la lengua tzeltal y diccionario de la misma, Chiapas, México.

Pitarch Ramón, Pedro (1996), Ch'ulel: una etnografía de las almas tzeltales, México: Fondo de Cultura Económica.

Recinos, Adrián (Editor) (1978), Popol Vuh. Las antiguas historias del Quiché, San José: Educa.

Ruz, Mario Humberto (1992), Copanaguastla en un espejo. Un pueblo tzeltal en el virreinato, México: Consejo Nacional para la Cultura y las Artes-Instituto Nacional Indigenista.

Slocum, Mariana C., Florencia L. Gerdel, y Manuel Cruz Aguilar (1999), Diccionario tzeltal de Bachajón, Chiapas, Serie de Vocabularios Indígenas "Mariano Silva y Aceves", núm. 40, México: Instituto Lingüístico de Verano.

Tedlock, Barbara (2002), El tiempo y los mayas del altiplano, Guatemala: Ediciones Yaxte'.

Tejada Buscayrol, Mario (2005), Notas de campo de Santa Eulalia, Manuscrito inédito. 


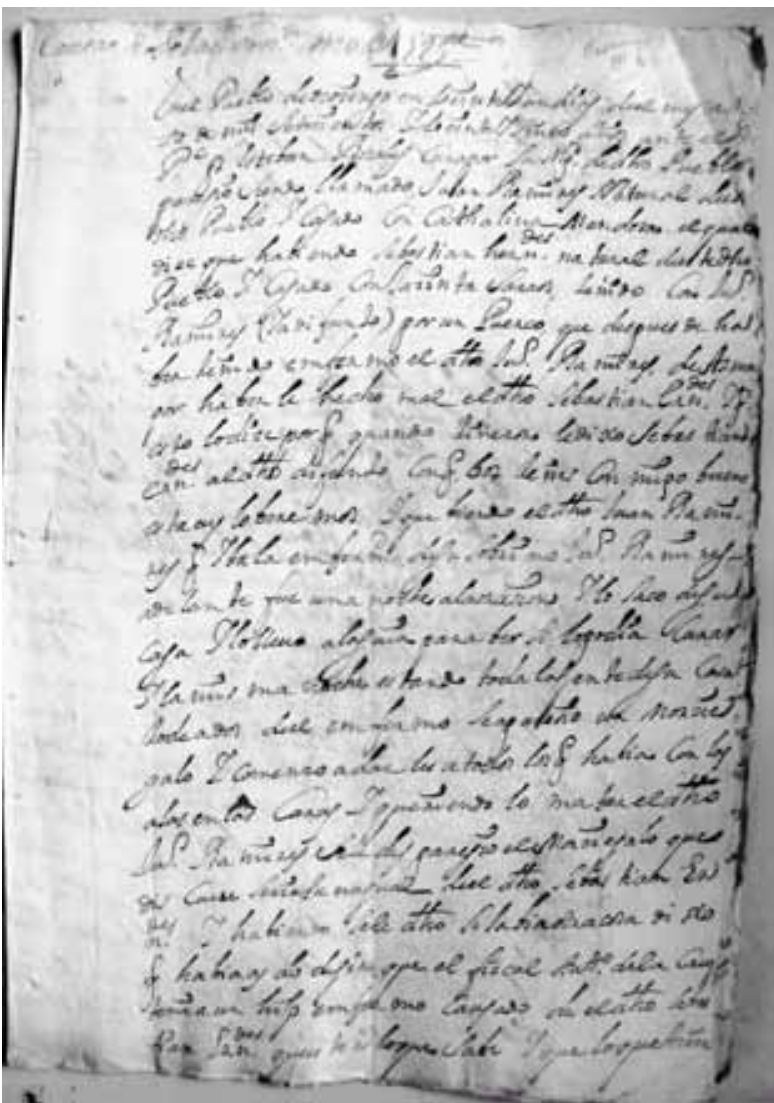

(1)

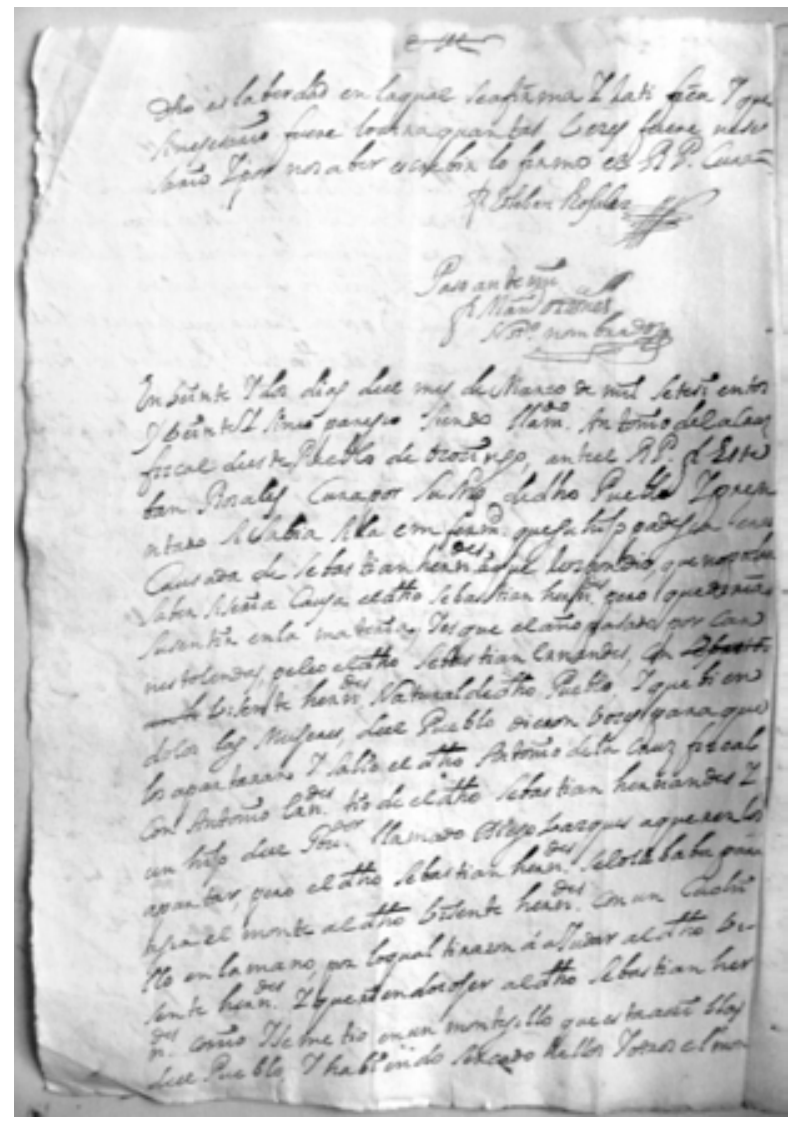

(2) 


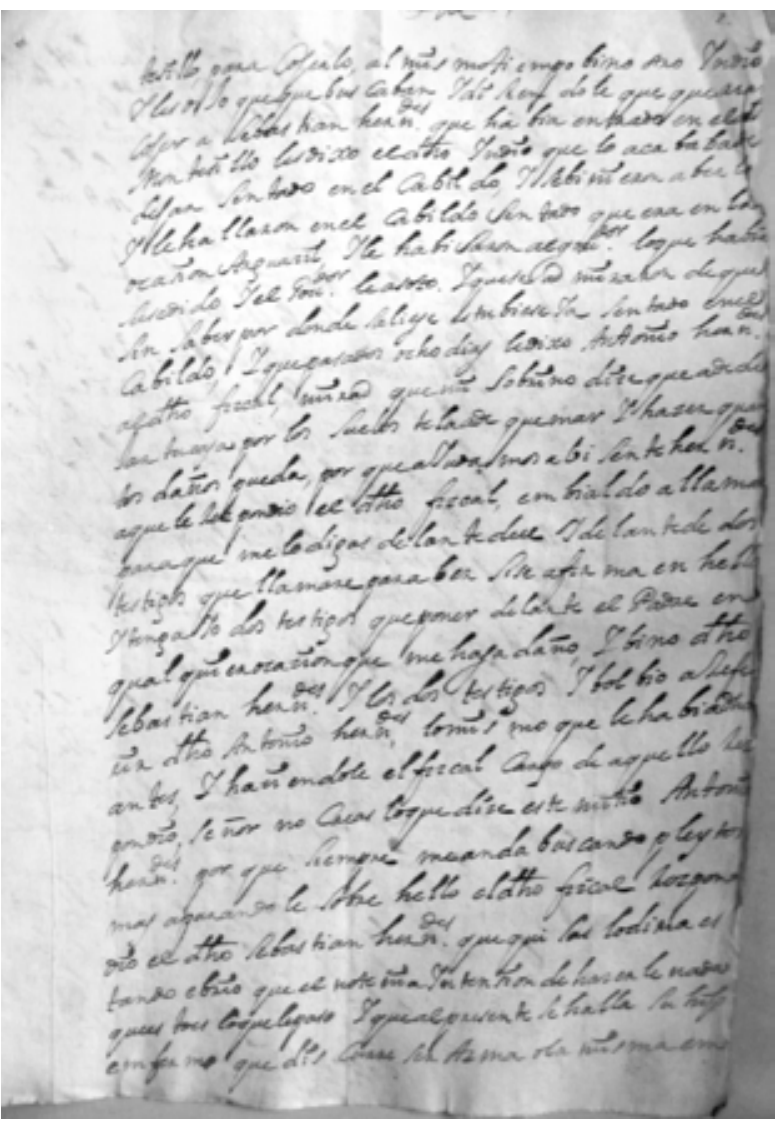

(3)

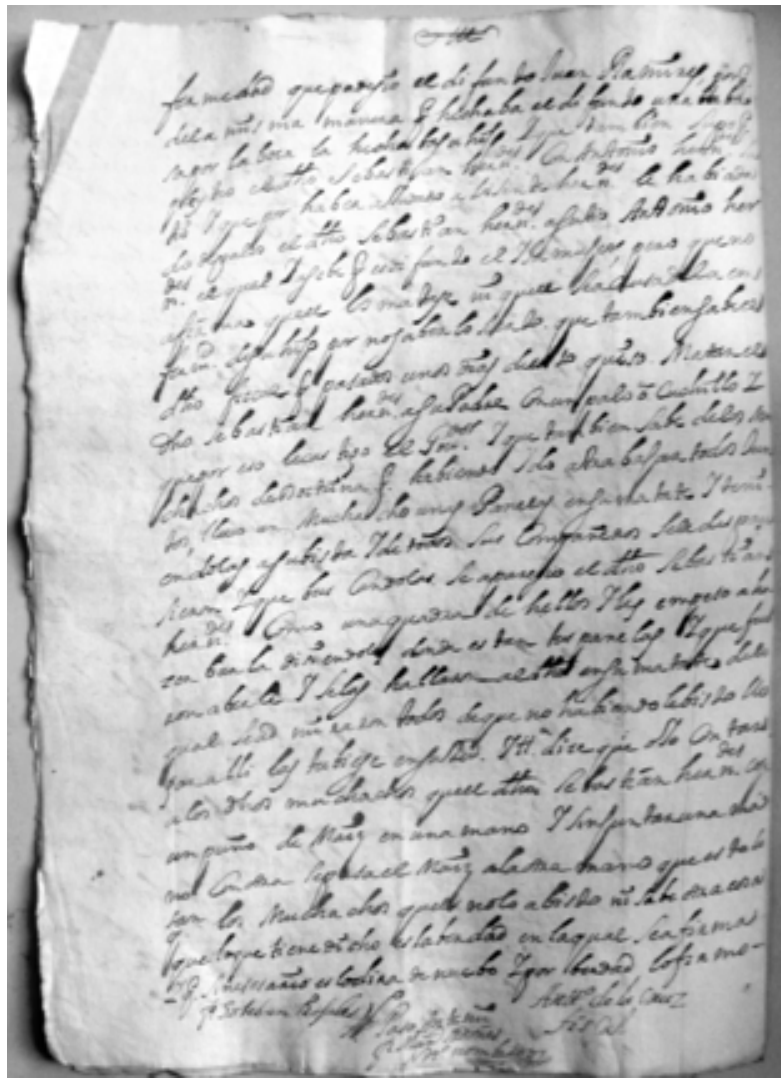

(4) 


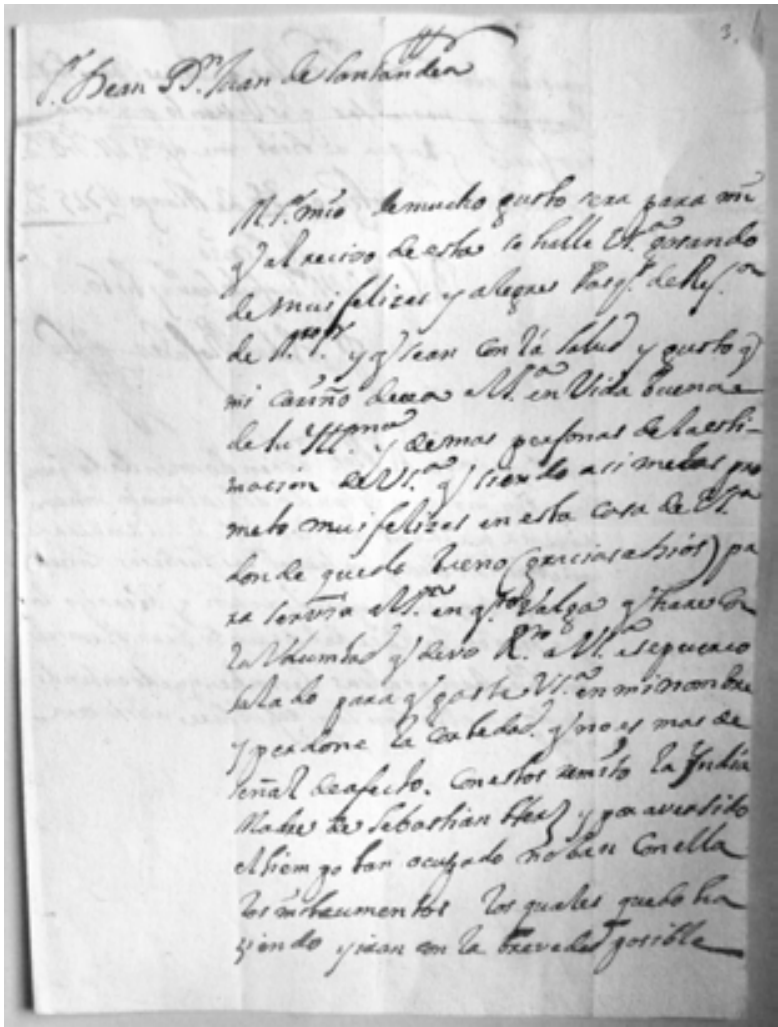

(5)

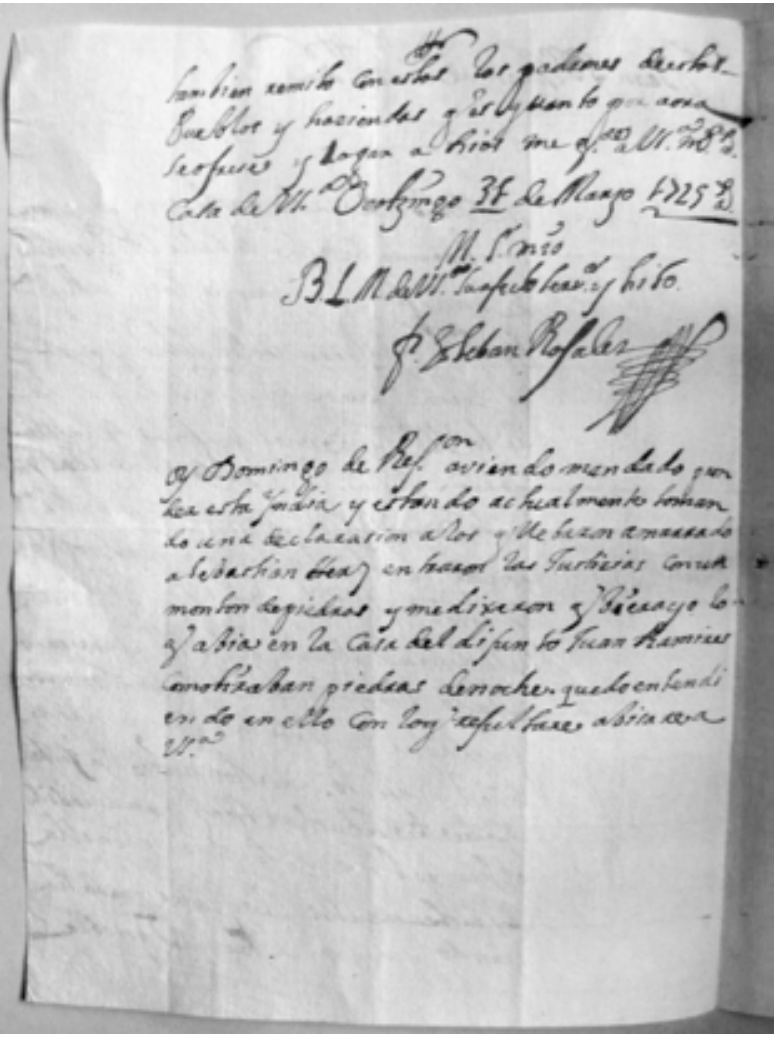

(6) 


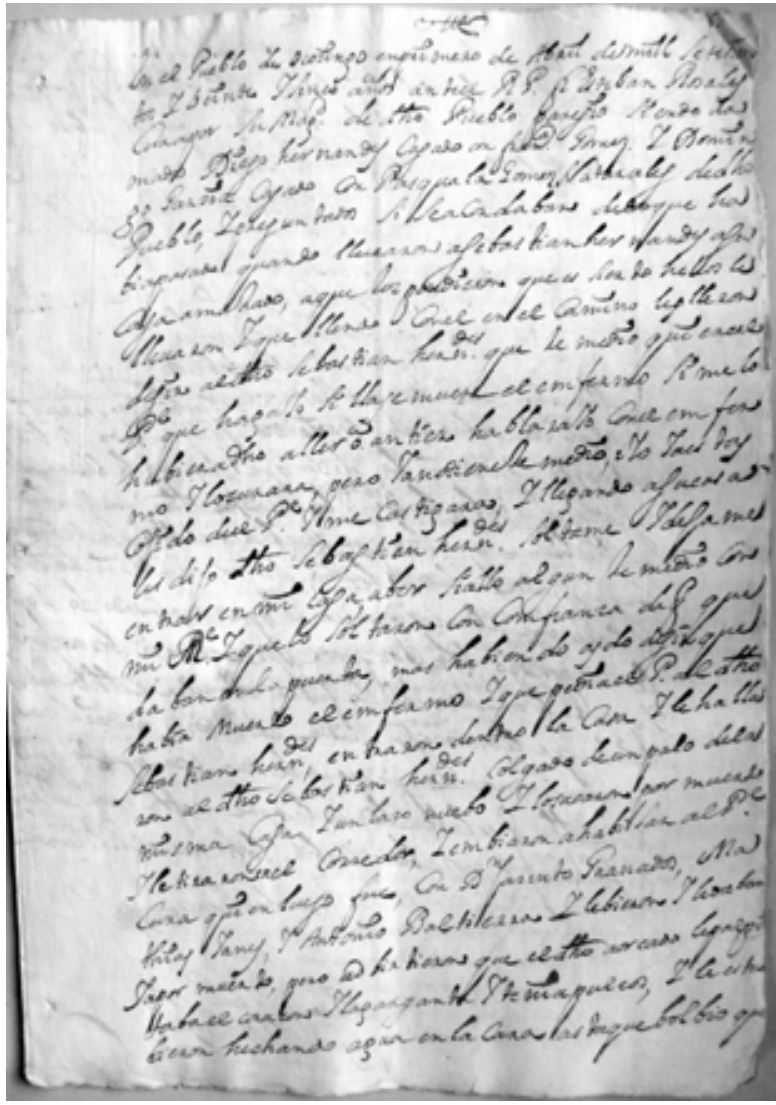

(7)

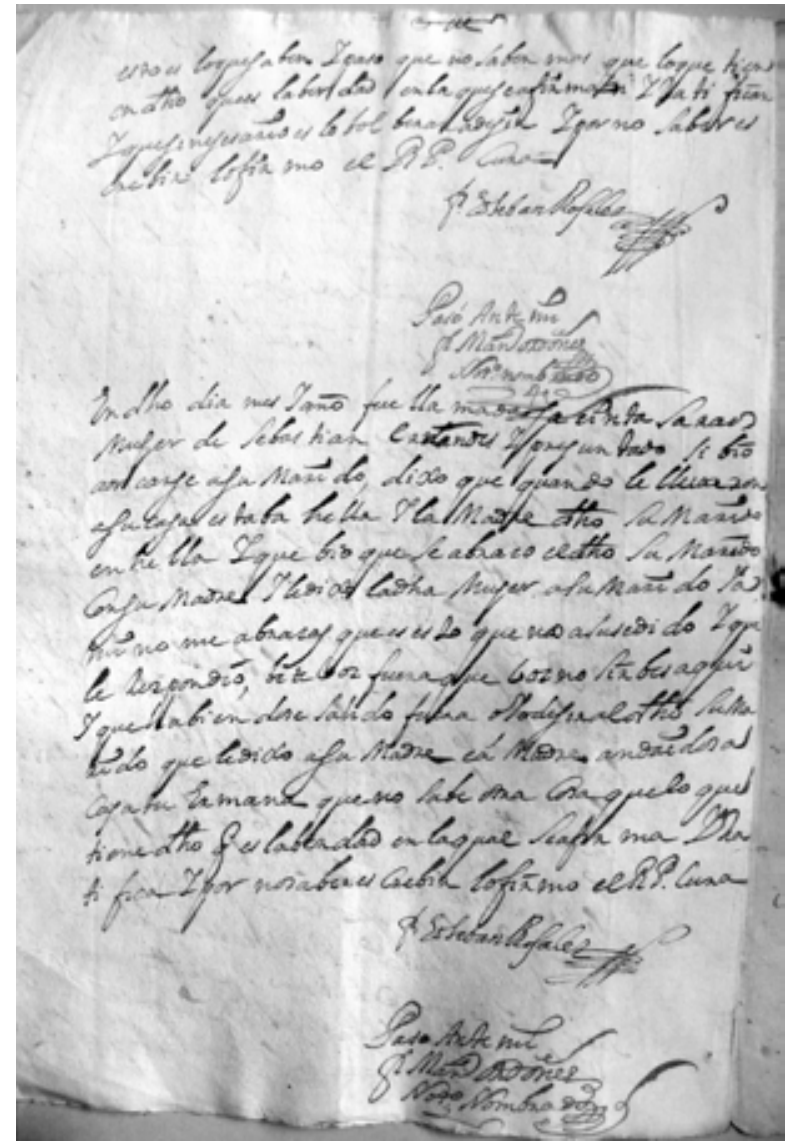

(8) 


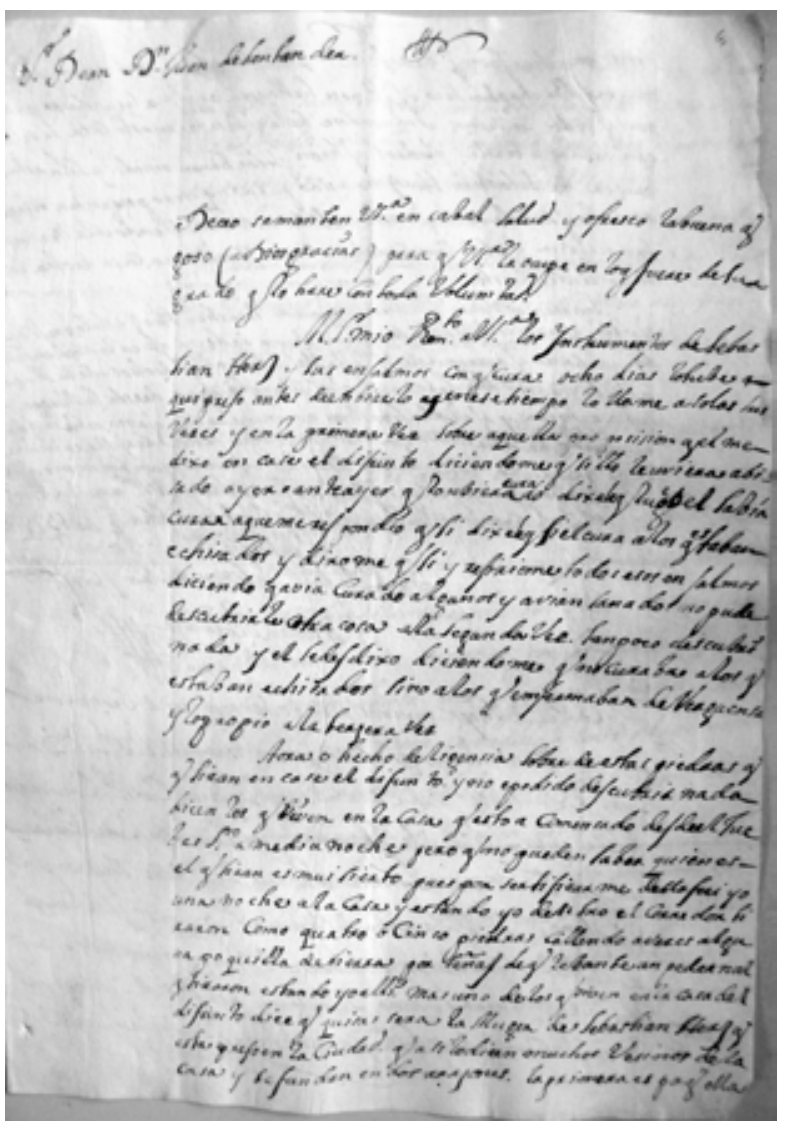

(9)

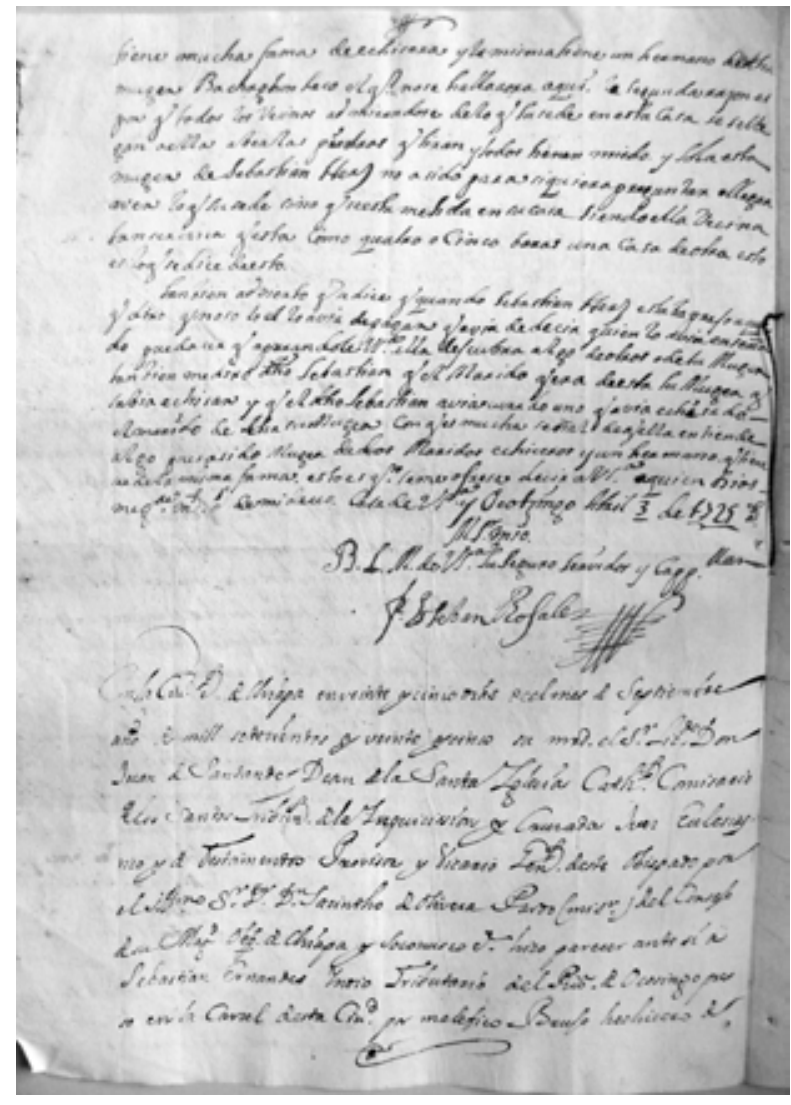

(10) 


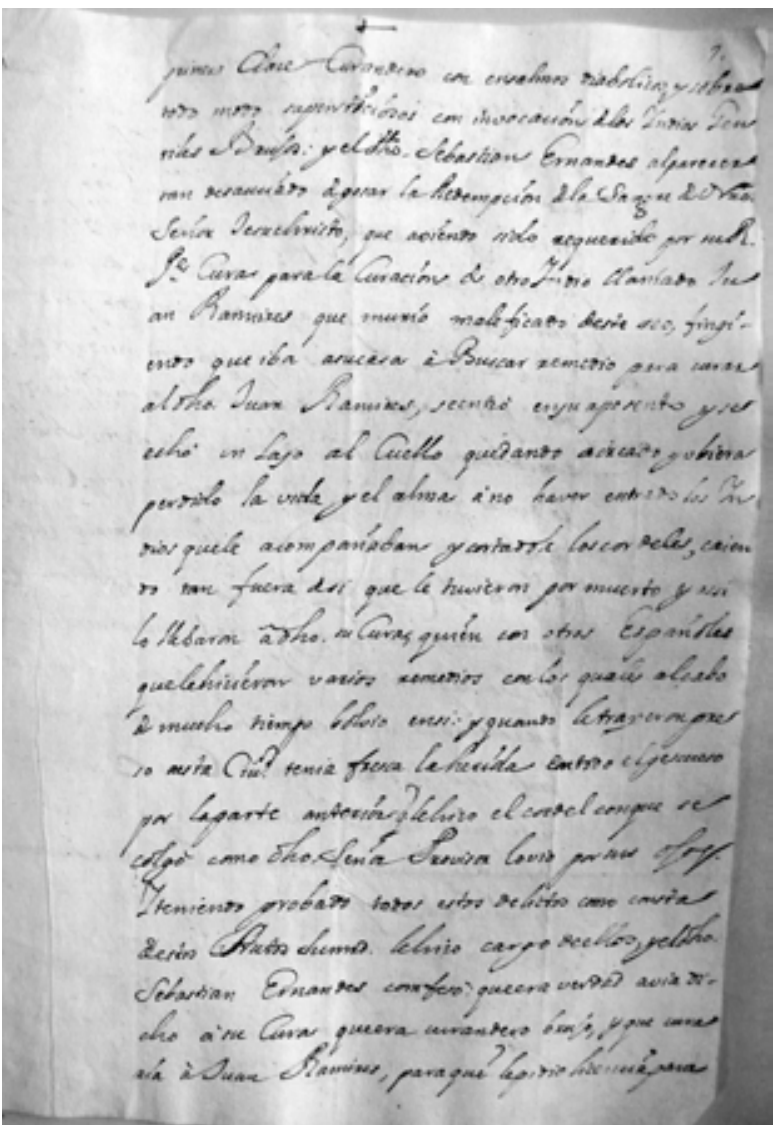

(11)

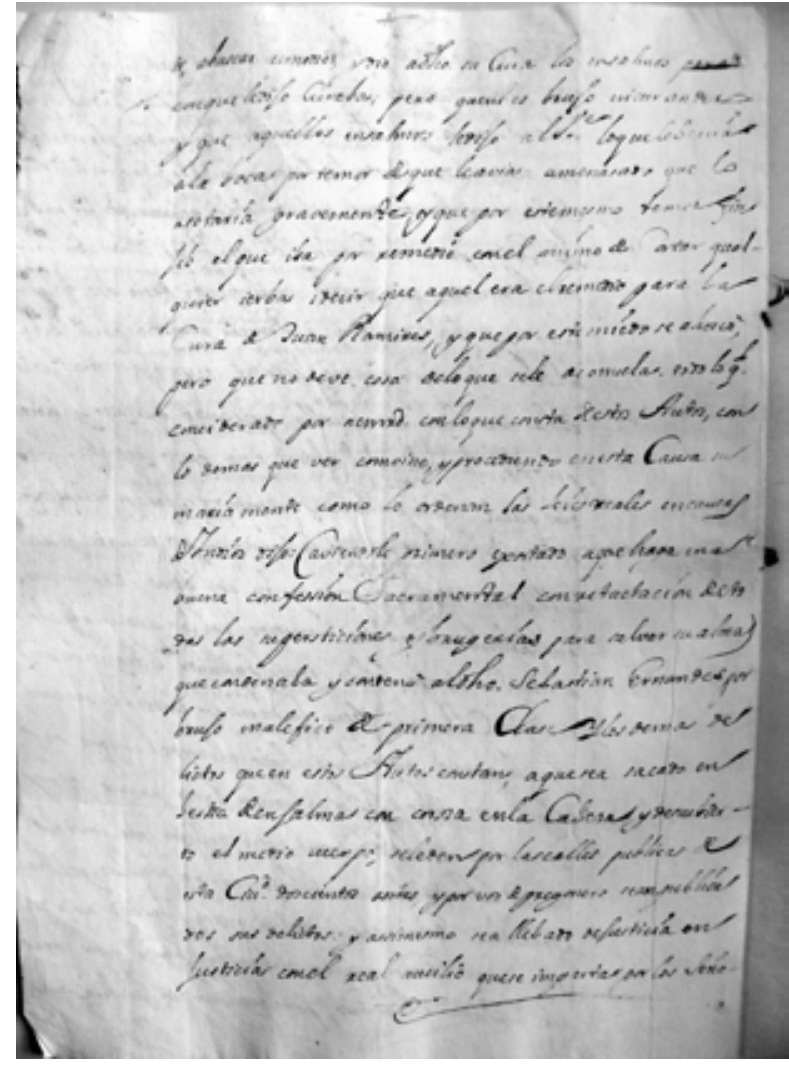

(12) 


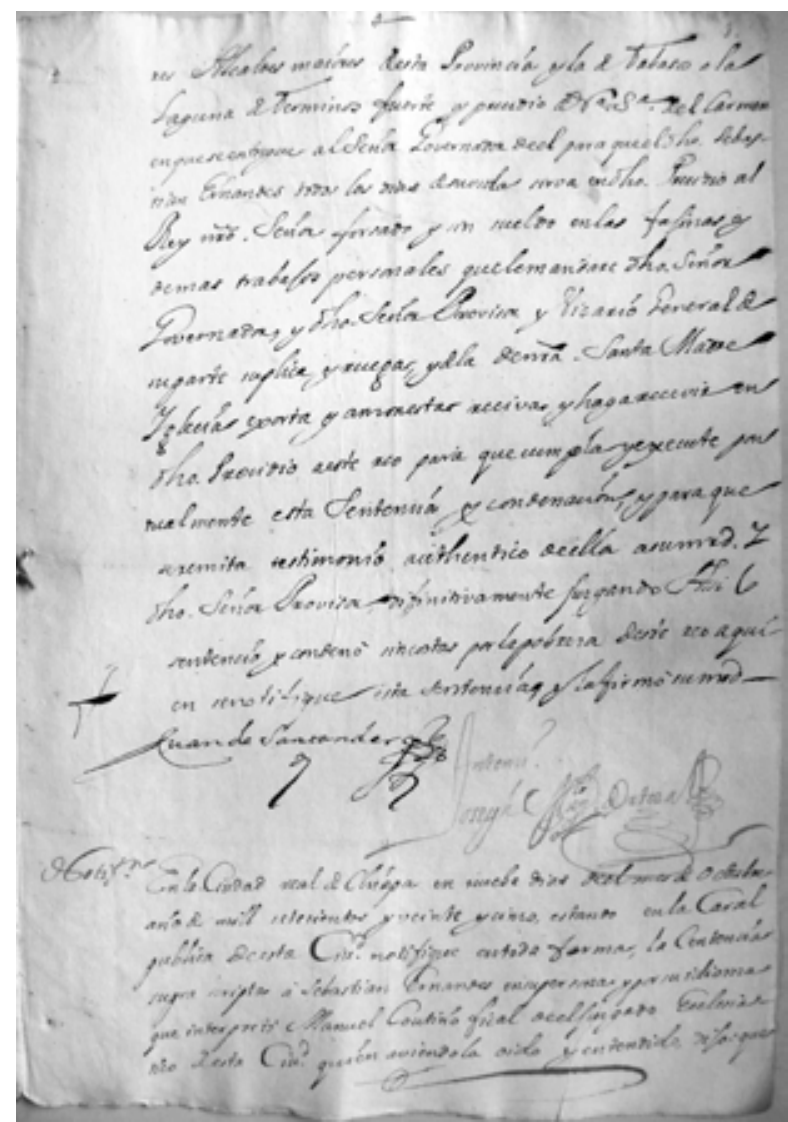

(13)

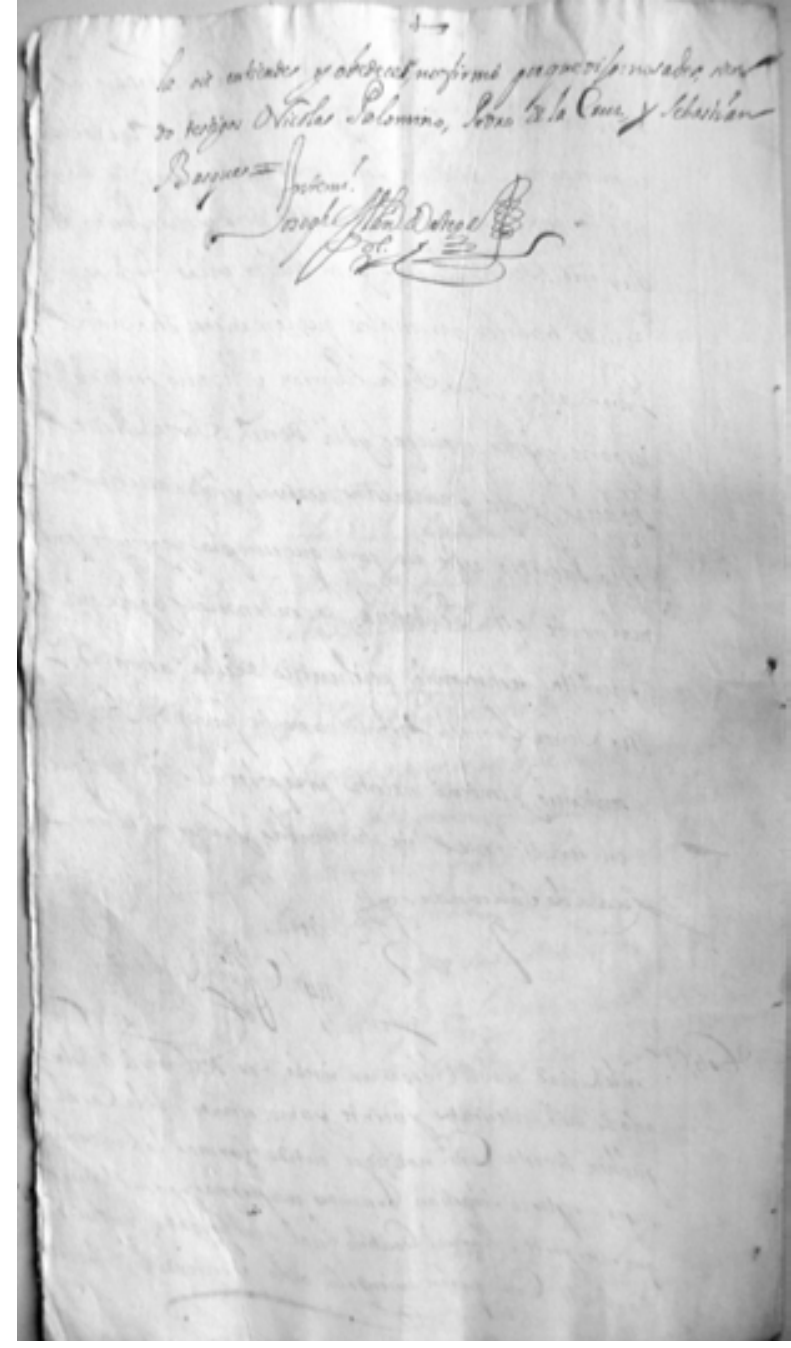

(14) 


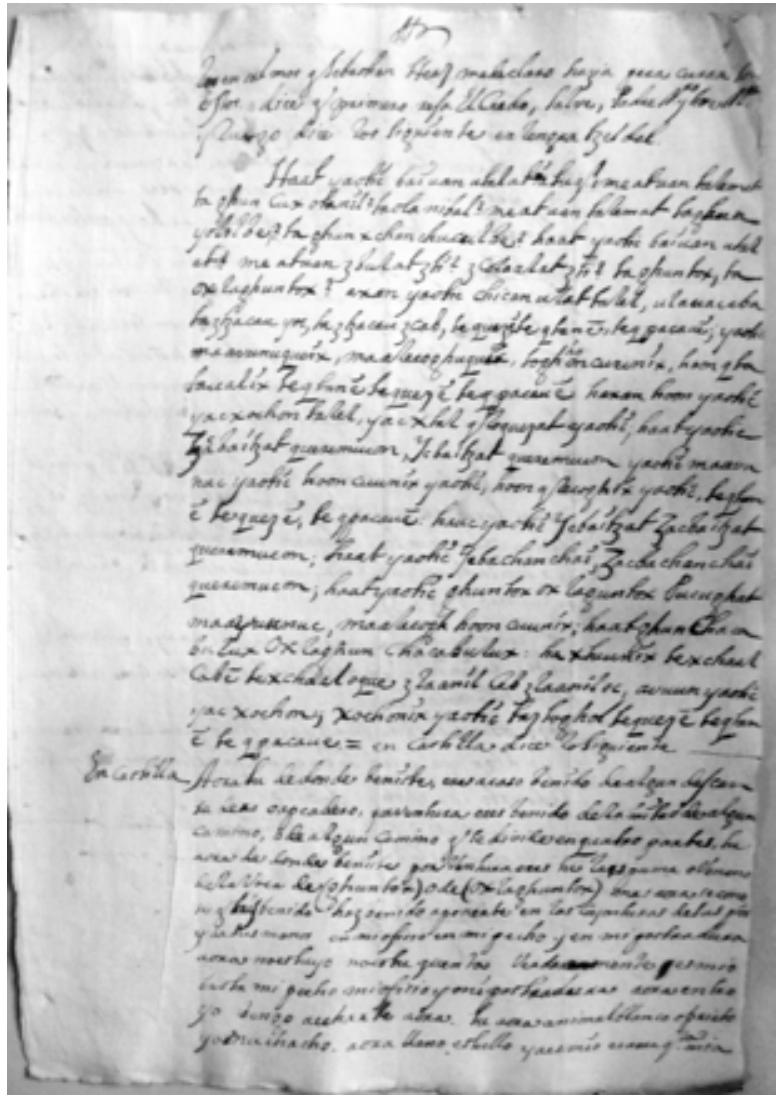

(15)

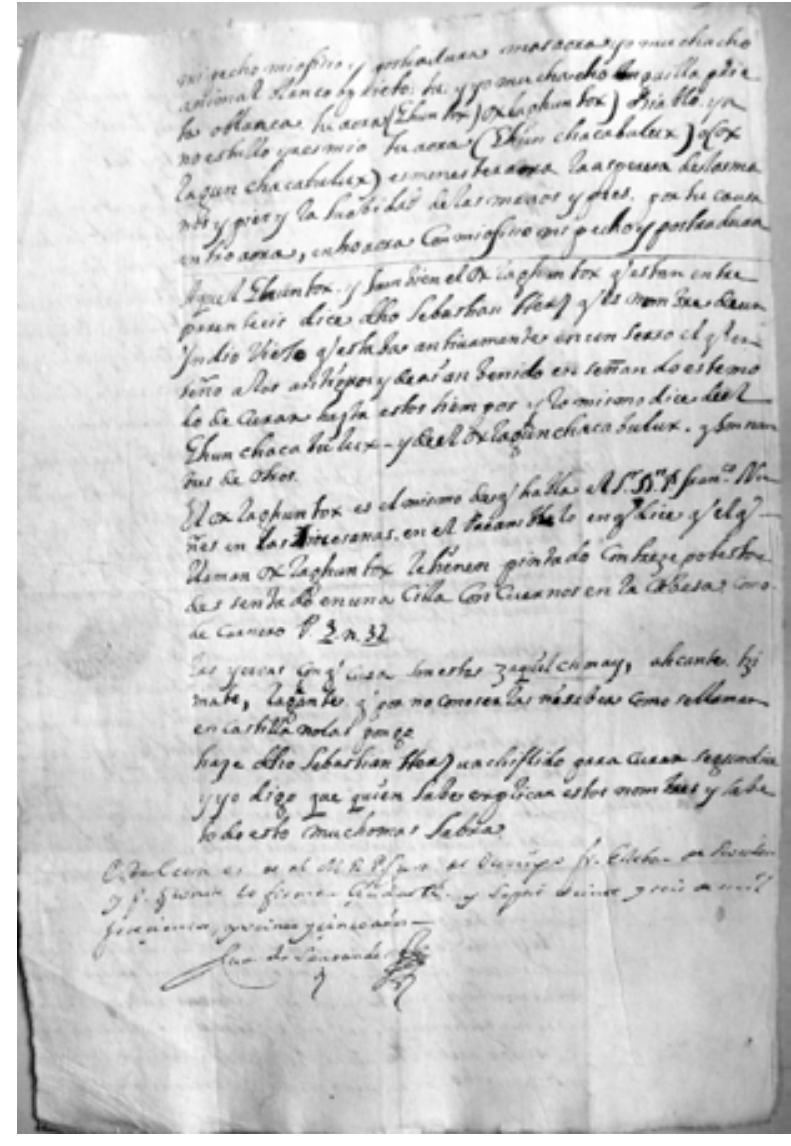

(16) 